\title{
Capsule Commentary on Zuchowski et al., Challenges in Referral Communication Between VHA Primary Care and Specialty Care
}

\author{
Samantha L. Solimeo, PhD, MPH \\ Department of Veterans Affairs VISN 23 Patient Aligned Care Team Demonstration Laboratory and the Center for Comprehensive Access \& \\ Delivery Research and Evaluation (CADRE), lowa City, IA, USA.
}

J Gen Intern Med 30(3):344

DOI: $10.1007 / \mathrm{s} 11606-014-3146-9$

(C) Society of General Internal Medicine 2014

$\mathrm{P}$ otential improvements in clinical outcomes and reduced costs resulting from high quality coordination between primary care (PCP) and specialty care providers (SCP) will remain unrealized without substantive attention to the technological and interprofessional factors affecting continuity and co-management. ${ }^{1}$ Zuchowski and colleagues conducted this mixed method study to identify and understand PCPs' perceived barriers to communicating with SCPs in the nationally integrated VA health care system setting. ${ }^{2}$ Survey data collected from PCPs were used to evaluate variation in perceived ease of communication with SCPs by provider type. Qualitative interviews conducted with primary care medical home team members from the same region were analyzed to provide an explanatory framework for the quantitative findings. Survey data indicate that populationfocused SCPs were generally considered the easiest to communicate with, while those from surgical specialties were viewed by PCPs as the most difficult. Interview data identified two communication challenges: SCP rejection of PCP referral requests and PCP inability to communicate patientspecific scheduling needs effectively. ${ }^{2}$ Together, the overlapping samples illustrate general trends in communication barriers that persist despite VA's infrastructural and fiscal advantages over the private sector.

While the study design did not collect corresponding data from SCPs, validate SCP referral rejection rate, or examine SCP factors in delayed scheduling, extant literature indicates that these challenges are endemic and persistent. ${ }^{3-5}$ Data from PCPs in this study contribute evidence that electronic consults (i.e., e-consults) may ameliorate some of these challenges. E- consults can guide treatment decisions, identify information required for an effective specialty care visit, and perhaps most important, e-consults can foster interprofessional relationships between PCPs and the SCPs they may never meet in person, but on whose expertise they rely. As Taylor et al. suggest, partnered with attention to "... referral rate for primary care physicians, the number of referrals avoided through preconsult exchanges, and the number of referrals that are completed and not completed," 3 e-consults have the potential to reveal specific practice patterns associated with unsatisfactory referrals, thus moving us closer to identifying trigger points for enacting co-management in medical home settings.

Conflict of Interest: The author has no conflicts of interest related to this article.

Corresponding Author: Samantha L. Solimeo, PhD, MPH; Department of Veterans Affairs VISN 23 Patient Aligned Care Team Demonstration Laboratory and the Center for Comprehensive Access Delivery Research and Evaluation (CADRE), Iowa City VA Health Care System, Iowa City, IA 52246, USA (e-mail: Samantha.solimeo@va.gov).

\section{REFERENCES}

1. Greenberg JO, Barnett ML, Spinks MA, Dudley JC, Frolkis JP. The 'medical neighborhood': Integrating primary and specialty care for ambulatory patients. JAMA Intern Med. 2014;174:3.

2. Zuchowski JL, Rose DE, Hamilton AB, Stockdale SE, Meredith LS, Yano EM, Rubenstein LV, Cordasco KM. Challenges in Referral Communication Between VHA Primary Care and Specialty Care. J Gen Intern Med. 2014. doi:10.1007/s11606-014-3100-x.

3. Taylor EF, Lake T, Nysenbaum J, Peterson G, Meyers G. Coordinating Care in the Medical Neighborhood: Critical Components and Available Mechanisms. 2011. White Paper prepared by Mathematica for the Agency for Healthcare Research and Quality. AHRQ Publication No. 11-0064

4. Hysong SJ, Esquivel A, Sittig DF, Paul LA, Espadas D, Singh S, Singh H. Towards successful coordination of electronic health record-based referrals: A qualitative analysis. Implementation Science. 2011;6:84.

5. Mehrotra A, Forrest CB, Lin CY. Dropping the baton: Specialty Referrals in the United States. The Millbank Quarterly. 2011;89:1.

Published online December 23, 2014 